\title{
MULTI-FILAMENT PCSS MODULES TO REPLACE HIGH CURRENT PULSED POWER SWITCHES
}

\author{
F.J. Zutavern ${ }^{\xi}$, A. Mar, G.A. Vawter, \\ S.F. Glover, H.P. Hjalmarson, K.H. Greives \\ * Sandia National Laboratories, Albuquerque, NM 87185 USA
}

\section{Abstract}

\begin{abstract}
Methods of producing multiple current-sharing filaments (MCSF) in GaAs photoconductive semiconductor switches (PCSSs) have produced as many as 30 filaments per switch with a spacing of $330 \mu \mathrm{m}$. As the approaches to triggering and isolating MCSF mature, the replacement of high current, conventional pulsed power switches with banks of MCSF PCSSs capable of switching tens of kiloamps become feasible and economical. Multiple banks of MCSF PCSS can eventually produce optically controlled pulsed power systems with faster rise-times, shorter pulses, and higher peak powers using more economical switches with device lifetimes of over one million pulses.
\end{abstract}

This paper will report progress from three types of MCSF approaches: (1) line-of-sight (LOS) optics focused with cylindrical micro-lens arrays into bright narrow lines of light on the surface of the PCSS; (2) high-reflectivity dielectric optical masks which produce shadows of bright narrow lines of light on the surface of the PCSS; and (3) etched-steps in the surface of the PCSS to divide up the illuminating light and isolate the filaments. These approaches will be tested to switch 2-3 kA with three $1 \mathrm{~cm}$ square GaAs PCSS. Switching parameters, approximate device lifetimes, and current-sharing capability are being measured and evaluated for each of these triggering techniques. Many other approaches have been considered previously, and their potential for other specific applications will be discussed.

Increasing PCSS current density through the development of longer-lived PCSS with higher currents per filament is research that has continued in parallel with the multi-filament triggering work. This however is primarily an interface issue where the contact metal meets the GaAs. We will discuss new electrical contact geometries capable of reducing current crowding, thereby lowering the peak current density to enable higher total filament current. Theoretical bases for improving contacts will also be discussed.

\section{INTRODUCTION}

GaAs PCSS exhibit two very important properties at average electric fields above $4-6 \mathrm{kV} / \mathrm{cm}$. The first is avalanche carrier generation, which reduces their required trigger energy by several orders of magnitude, when compared to linear PCSS, in which only one electron-hole (e-h) pair is generated per absorbed photon.

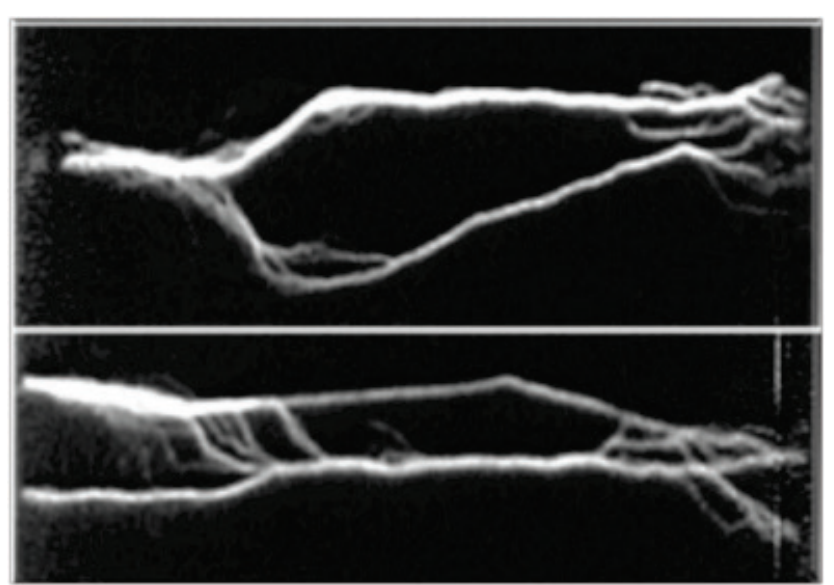

Figure 1. These pictures show $875 \mathrm{~nm}$ recombination radiation from current filaments in high gain GaAs PCSS that were $1.5 \mathrm{~cm}$ tall switching $40 \mathrm{kV}$ and $350 \mathrm{~A}$ for 10 ns. The filaments were initiated with a $1 \mathrm{~mm}$ diameter spot of $532 \mathrm{~nm}$ light near the right of each photo.

Above these threshold fields, low impedance circuits have produced as many as $10^{5}$ e-h pairs per absorbed photon, which explains why they are often called "high gain" PCSS. Avalanche carrier generation is also the reason GaAs PCSS remain in a low resistance state after the laser pulse is gone, when the circuit maintains an onstate field greater than this threshold across the PCSS. Hence, this field is often called the "lock-on" field.

The second important property exhibited by GaAs PCSS is that current forms in filaments when they are operated in the high gain mode (figure 1). Current filaments imply high current densities, and they are generally a property to be avoided, if possible. However, the first property, which allows very low energy triggering typically in the nanojoule to microjoule regime, is so important for many applications that it is worth dealing with current filamentation. By limiting the current per filament to $20 \mathrm{~A}$ and the current pulse width to 20 ns, high gain GaAs PCSS with extremely conductive contacts have demonstrated device lifetimes greater than $10^{8}$ pulses. Higher currents, longer pulses, and/or more resistive contacts produce shorter lifetimes, e.g. $50 \mathrm{~A}, 50$ ns with evaporated and annealed contacts typically produces several hundred to a few thousand shots.

A short pulse application that requires high voltage (HV) and only low current, or a few filaments, is a trigger for conventional pulsed power switches. Tests with a variety of electrically triggered $\mathrm{HV}$ spark gaps have

Sandia National Laboratories is a multi-program laboratory managed and operated by Sandia Corporation, a wholly owned subsidiary of Lockheed Martin Corporation, for the U.SDOE's National Nuclear Security Admin. under contract DE-AC04-94AL85000, SAND \# 2013-5438C. This work supported by a DTRA/MOU and the Laboratory Directed Research and Development Program at SNL.

U.S. Government work not protected by U.S. copyright 
shown that a low current PCSS in close proximity to the trigger pin can produce lower jitter switching over a larger operating voltage range than the best reported results with conventional $50 \Omega \mathrm{HV}$ trigger systems [1]. PCSS trigger generators, such as the one shown in figure 2 for a $200 \mathrm{kV}$ linear transformer driver (LTD) only require 1-5 filaments depending on the PCSS lifetime requirements.

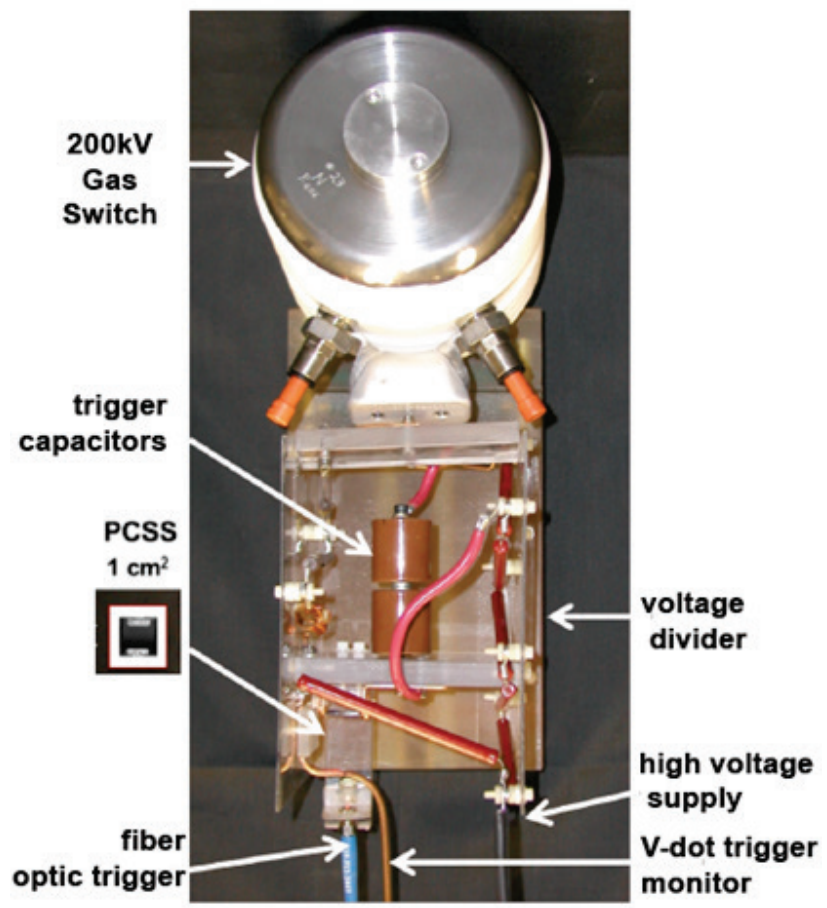

Figure 2. The PCSS trigger for a $200 \mathrm{kV}$ LTD switch is built into the LTD module. Its close proximity eliminates the need for a HV $50 \Omega$ trigger cable. Trigger energy is derived from the power to the module capacitors.

To switch much higher currents and replace instead of trigger conventional pulsed power switches, the location of the filaments must be precisely controlled to produce MCSF. As shown in figure 1, filaments in GaAs form like lightning in branches which intersect and to not divide the current uniformly. Our highest voltage and current results were produced using a bank of 6 2-inch diameter GaAs wafers to switch $210 \mathrm{kV}$ and $8 \mathrm{kA}$ [2]. The filaments were controlled by focusing the trigger into straight lines onto the GaAs wafers with 180 1-mm diameter glass rods, implying $44 \mathrm{~A} /$ filament, if all trigger light lines produced filaments. However, this was not a practical, scalable approach to high current switching.

There are many possible ways to trigger MCSF in GaAs, some of which have been tabulated in a previous paper [3]. Edge-emitting semiconductor lasers produce very sharp lines and have triggered filaments with less than $100 \mathrm{~nJ}$. The best estimate of the intensity required to trigger filaments, $80 \mathrm{~kW} / \mathrm{cm}^{2}$, is from edge-emitting laser triggering. This was based on $100 \mathrm{~nJ}$ in $5 \mathrm{~ns}$ focused into $10 \mu \mathrm{m}$ X $2.5 \mathrm{~mm}$ lines. However, large stacks of edgeemitting lasers do not provide the uniform and reliable lasing required to trigger large numbers of MCSF. The stacking process is a tedious low-yield approach. One poorly performing laser in the edge emitting array can destroy a PCSS in only a few shots. This paper reports the development of GaAs PCSS modules using two of the approaches to triggering MCSF listed in reference 3 and one new approach described in subsequent sections. Parallel efforts to increase device lifetime (or equivalently current per filament) by improving contact fabrication techniques are also described. Finally, modeling efforts to explain and simulate these unusual properties of GaAs are similar to those used to model breakdown discussed in another paper in these proceedings [4].

\section{EXPERIMENTAL SETUP}

Contact development and new fabrication techniques are normally tested with small devices, a few hundred microns long at a few hundred Volts or $10-100 \mathrm{kV} / \mathrm{cm}$. Switches for higher voltage applications are tested with much larger devices ranging from 1-15 mm long at up to $100 \mathrm{kV}$ or $10-70 \mathrm{kV} / \mathrm{cm}$. Both sizes of switches are typically pulse charged in the 1-50 $\mu$ s regime, although DC charging has been demonstrated at reduced fields for nuetron irradiated and longer switches [1]. Figure 3 shows the high voltage test bed that we are presently using to test triggering of MCSF. Since trigger testing is not always succesful, a low current circuit is used to test the initial alignment with new devices. The schematic for the low current circuit is shown in figure 4 . The switch mount for this cicuit consists of three plates to produce the same fields at the PCSS as the Blumlein, with much less capacitance. Current is adjusted by changing the pulse charged capacitors.

Once reliable MCSF triggering is achieved, the high current Blumlein is used to carry $2.5 \mathrm{kA}$. The impedances of the Blumlein are balanced to compensate for the onstate voltage across the PCSS. Both circuits are pulse charged $(\sim 10 \mu \mathrm{s})$ with a trigatron and a large inductor.

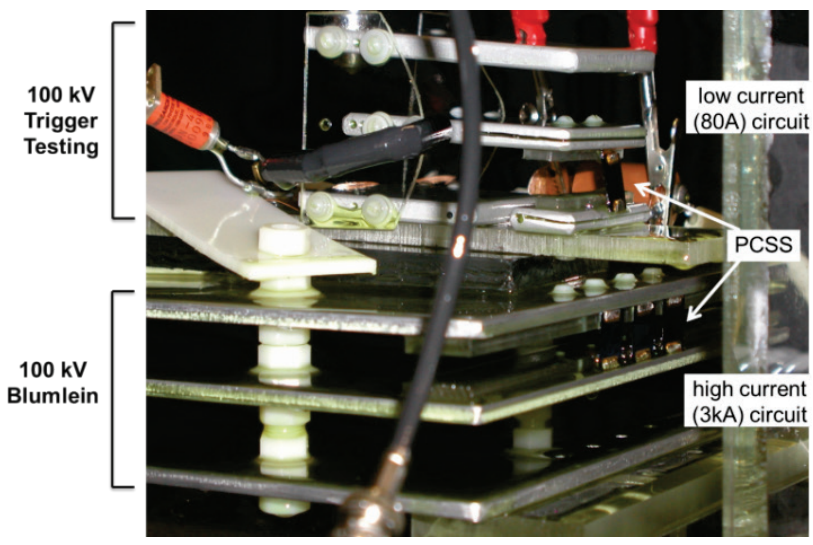

Figure 3. A high current $(2.5 \mathrm{kA}) 40 \Omega$ Blumlein (bottom) and a low current (20-80A) capacitive discharge circuit (top) are shown. One PCSS is mounted in the top circuit and three PCSSs are mounted on the Blumlein. 
Both circuits are suspended from an oil tank cover which lifts them out of the oil when it is raised. The circuits and optical path are shown in a diagram in figure 4. The laser approaches the PCSS through a Lucite box which displaces the oil so the beam only passes through a few centimeters of oil.

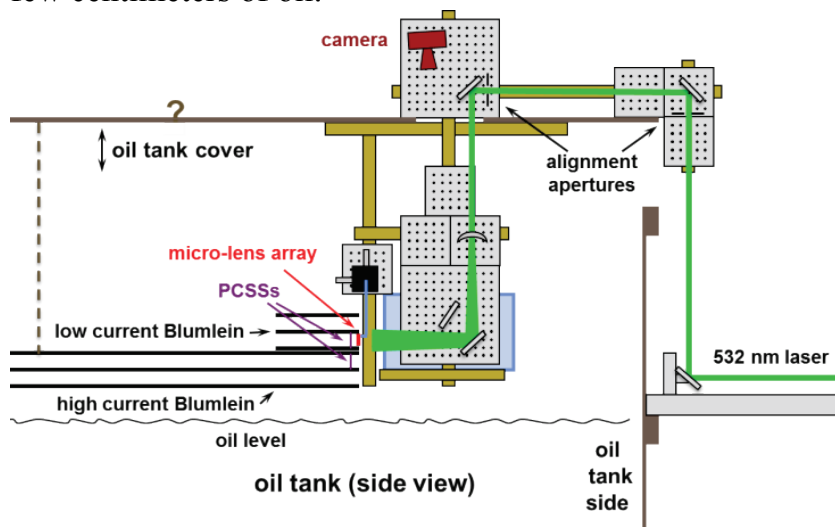

Figure 4. A diagram of the testbed shows the circuits suspended from the oil tank cover. Approximate alignment is maintained as the cover is raised.

\section{CYLINDRICAL $\mu$-LENS ARRAYS}

Optics that modulate the intensity of a laser beam into linear arrays of straight lines are probably the simplest optical concept for producing MCSF. However, most approaches have the disadvantage of requiring diffractionlimited LOS optics and a high quality laser beam. There are two important additional issues associated with focusing a laser beam into many parallel lines in transformer oil. First, the refractive index of oil is approximately the same as the common optical glasses and plastics that are used for optics. Thus, the effective focal lengths for optics or optical paths immersed in oil are much longer than they would be in air. Commercialoff-the-shelf (COTS) cylindrical $\mu$-lens have a moderate range of parameters. However, their focal lengths under oil are several centimeters or longer.

The second issue is caused by the spacing of the lenses, which also determines the desired spacing of the trigger lines. Since MCSF spacing of $300 \mu \mathrm{m}$ has been demonstrated with stacked edge emitting lasers, a similar spacing is desired for the array of cylindrical lenses. This small spacing which is the minimum aperture width in the system and the relatively long focal lengths of the available $\mu$-lens arrays under oil produce a diffraction limit $(\sim 100 \mu \mathrm{m})$ that is larger than the desired width of the optical trigger lines. Custom arrays can be fabricated with shorter focal lengths or from glasses or plastics with larger refractive indexes to reduce the effective focal lengths under oil. However, these are too expensive to purchase until the final lens parameters have been determined. To reduce diffraction limited line widths with COTS optics, $2 \mu$-lens arrays were used in series to optimize the parameters for MCSF triggering.

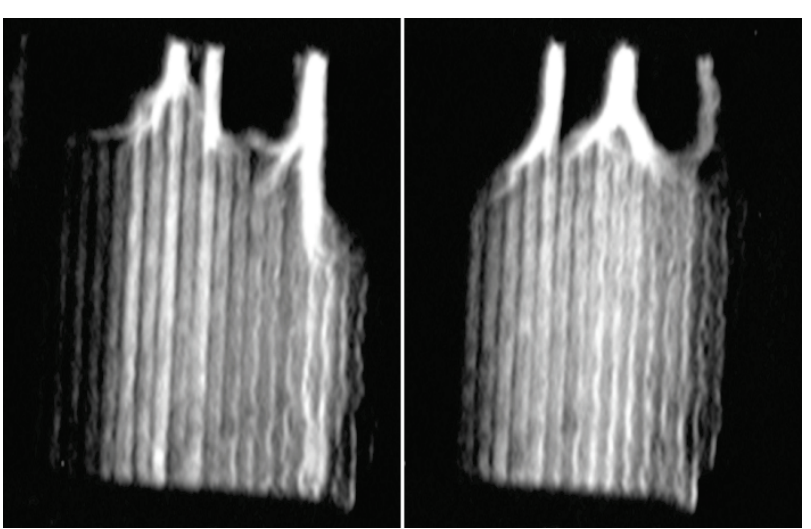

Figure 5. Examples of filaments produced with $1 \mathrm{~cm}$ long GaAs PCSS and a pair of cylindrical $\mu$-lens arrays show reasonable current sharing except near the top.

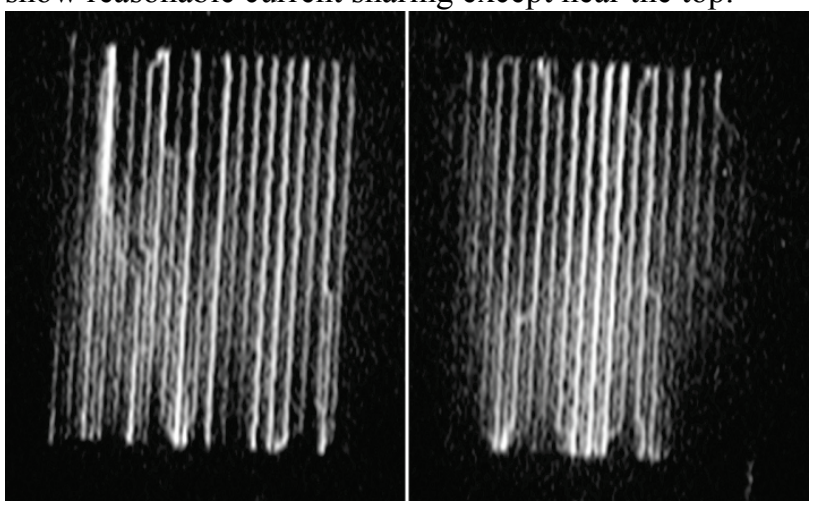

Figure 6. Current sharing filaments were produced with a larger laser beam and improved alignment using a pair of cylindrical $\mu$-lens arrays on a $1 \mathrm{~cm}$ long GaAs PCSS.

Figure 5 shows examples of initial tests using a $1 \mathrm{~cm}$ long GaAs PCSS in the low current test circuit with two cylindrical $\mu$-lens arrays producing multiple filaments that shared current part way across the PCSS. Adjusting the position of the laser and the focal position of the $\mu$-lens arrays moved the position of the filaments but did not produce MCSF that crossed the entire gap. The positioning of the $\mu$-lens arrays was improved by assembling a translation stage (metal) holding the plastic arrays that could be mounted above the switch on a ground plane, which could be adjusted without raising the cover of the oil tank. It was also observed that the PCSS was tilted slightly relative to the beam path, so it wasn't parallel to the focal plane. Adjusting the tilt and fine tuning the position of the $\mu$-lens arrays produced the images shown in figure 6 . While these MCSF are not perfect they are much improved over figure 5. The next step in optimizing the filaments is to use a refractive beam shaper to produce a more flat-topped laser profile (presently Gaussian) to improve the laser uniformity over the entire wafer and reduce the optical trigger energy which is presently $\sim 2 \mathrm{~mJ}$. Further improvement will also be achieved when a higher quality, shorter focal length, glass, $\mu$-lens array is specified, procured, and installed. 


\section{OPTICAL MASKS}

Optical masks may be the simplest and least expensive approach to producing MCSF in a GaAs PCSS. If they are patterned onto the surface of the PCSS during the contact fabrication procedure, their cost would be a small increase and the optical constraints would be greatly reduced. MCSF could be triggered with uniform light sources from fiber optic bundles beam homogenizers, and LOS optics would not be required. Their main drawback is the light that would be lost in the mask. Some of this optical energy could be recovered, by combining this approach with one that modulates the optical intensity into the mask which would still improve the MCSF by producing narrower lines of light with greater extinction between the lines.

After testing the voltage hold-off for an insulating masking material on GaAs, uniform optical illumination was used to trigger filaments with images shown in figure 7. The image on the right shows promise. Results may be improved with more uniform illumination or masking. The image on the left had the narrowest line widths and was expected to produce the best results. However, microscopic examination following the tests showed that the masking material was no longer attached to the surface of the GaAs. The only remaining structures were vertical lines from etching the device to (ironically) improve adhesion of the mask. The fact that a few very straight filaments were observed, presumably caused by the modest $0.5 \mu \mathrm{m}$ deep etches, led us to consider deeply etched valleys and ridges as an approach to masking and isolating the filaments.

\section{ETCHED RIDGES AND VALLEYS}

Etched ridges and valleys (figure 8) could potentially produce filaments in 2 planes parallel to the surface. The valleys would physically isolate the filaments that form on the ridges and the ridges would absorb the illuminating light masking the filaments forming in the valleys.

The impact of surface roughness in the valleys on filament formation is not known and must be tested.

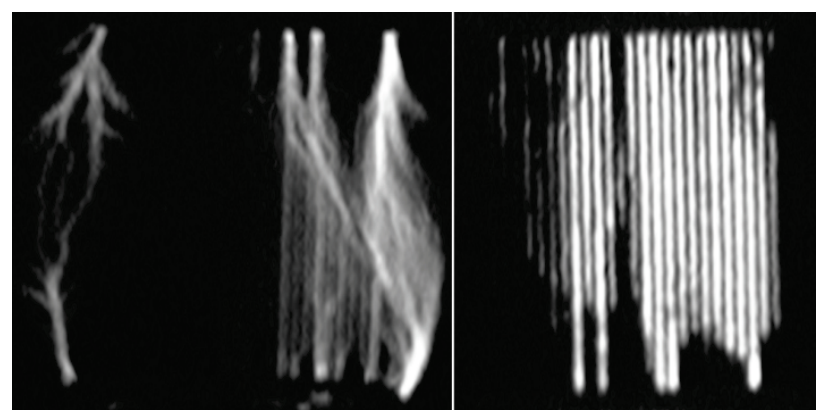

Figure 7. These filaments were produced with insulating optical masks patterned on the surface of the PCSSs. Both images had $300 \mu \mathrm{m}$ spacing, $20 \mu \mathrm{m}$ wide (left) and $80 \mu \mathrm{m}$ wide (right). The mask on the left did not adhere.

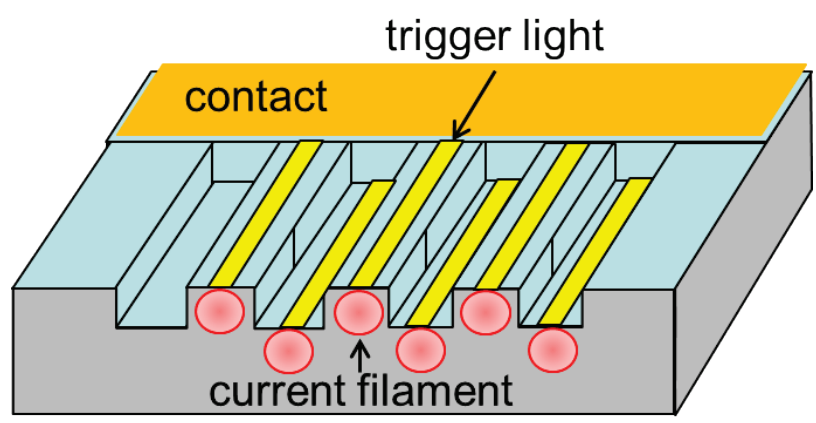

Figure 8. This diagram illustrates the ridge and valley concept near a contact on the surface of a PCSS. Etched steps $\sim 25 \mu \mathrm{m}$ deep will isolate the filaments that form on the ridges and mask the filaments that from in the valleys.

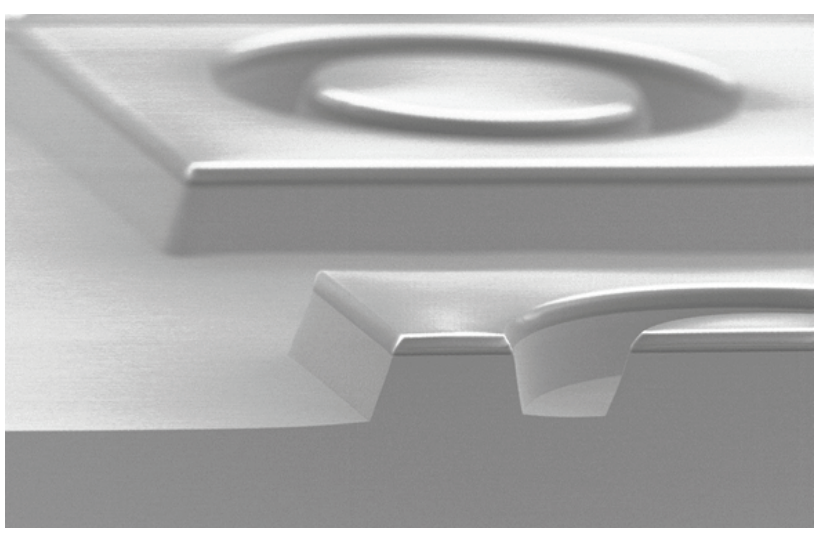

Figure 9. A scanning electron micrograph shows results from reactive ion etch forming a $\sim 40$ wide valley

The $\mu$-lens arrays discussed previously will be used to initiate filaments on only the ridges and only the valleys to discern the differences in filament formation. Figure 9 shows an image of preliminary etch testing to determine the etch depths that are practical.

\section{DEVICE LIFETIME}

\section{A. Improved contacts.}

To date, most PCSSs have been fabricated with relatively thin electrical contact metallization, similar to that shown in the top of figure 10. Evident degradation of contacts at end-of-life has led our team to explore thicker contacts with improved thermal conductance at the leading edge of the metal (figure 10 middle) and novel vertical contacts (figure 10 bottom), which are expected to distribute the filament current over a larger contact junction area, reduce the current density, and improve shot life. These studies are in progress.

\section{$B$. Trigger wavelength dependence.}

Because the bandgap energy wavelength of GaAs is about $\sim 880 \mathrm{~nm}$, triggering with $\mathrm{Nd}: \mathrm{YAG}$ lasers is commonly done with $532 \mathrm{~nm}$ frequency-doubling. $532 \mathrm{~nm}$ trigger light is strongly absorbed at the surface of the $\mathrm{GaAs}$ within a micrometer of the surface, and triggers filaments very efficiently. 

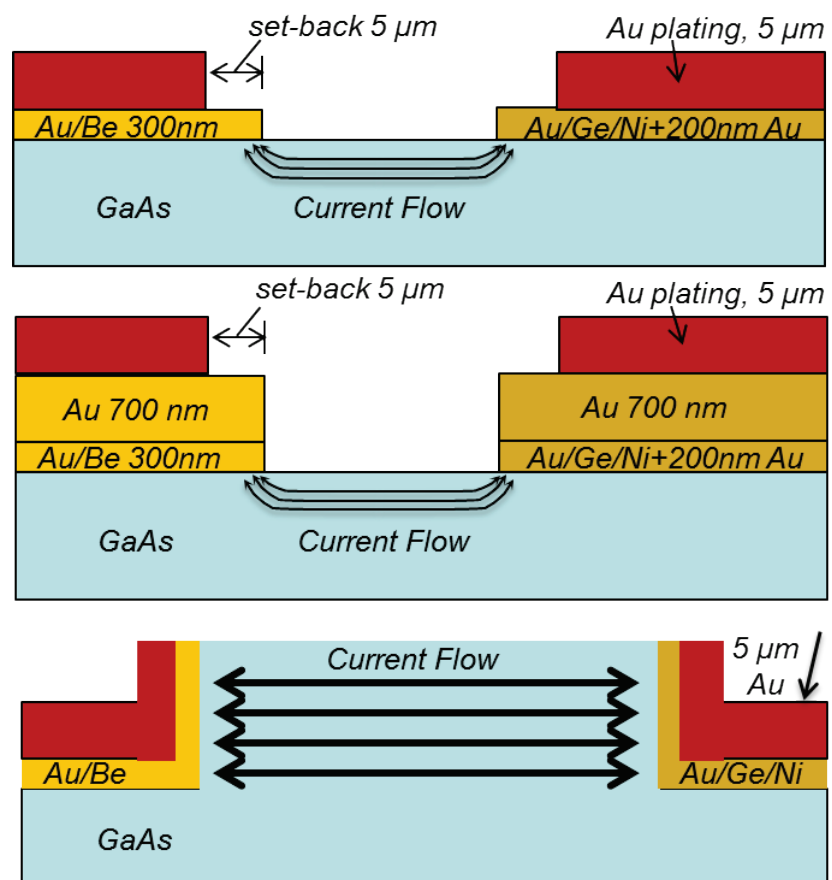

Figure 10. Diagrams of PCSS contacts show our present contacts (top), a plan to thicken the exposed edge of the gold under-layer(middle), and a new approach to eliminate the current pinching (bottom).

At $1064 \mathrm{~nm}$, weak absorption of trigger light occurs through EL2 defect sites that provide a midband energy state. On the order of 10x greater energy is required to initiate filaments compared to $532 \mathrm{~nm}$ operation, however this is partially offset by the conversion inefficiency incurred in the frequency doubling process. The $1064 \mathrm{~nm}$ trigger light is absorbed deeply through the thickness of the bulk GaAs.

We undertook a study of switch lifetime performance comparing triggering at the different wavelengths. Beam profiling was used to carefully match the optical spot sizes in the switch gap to ensure valid comparisons. The switch connected to a capacitive discharge (CDU) circuit that resulted in current pulses of nominally $100 \mathrm{~A}$ peak. Figure 11 shows the measured peak discharge current vs. shot number for switches triggered at $532 \mathrm{~nm}$ vs. $1064 \mathrm{~nm}$. The 532nm triggered PCSS switches higher peak current initially, but the $1064 \mathrm{~nm}$ triggered switch surpasses it after $\sim 200$ shots and continues for $\sim>2 \mathrm{x}$ greater number of shots before switch failure occurs.

\section{Optical trigger energy dependence.}

It has been observed through imagery of the current filaments that their structure seems to vary with the amount of trigger light applied. This is our first effort to quantify that behavior in terms of switch lifetime performance. During this test, we imaged the resulting filaments. Figure 12 shows the images for 4 consecutive firings early in each test for the same camera gain. Using computerized image analysis, we calculated the mean peak/average brightness ratios for the different firing sequences, to infer the peak spatial current density from the filament brightness in the images.

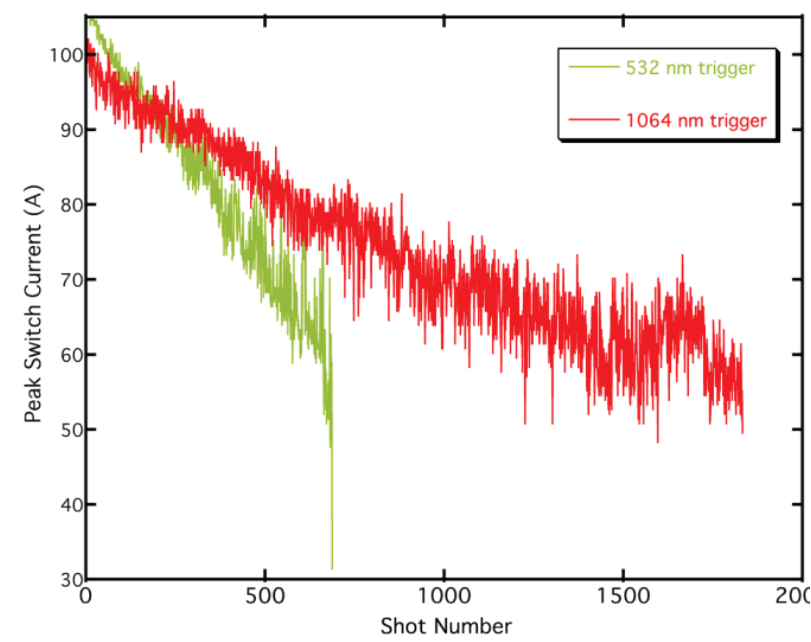

Figure 11. PCSS lifetest - peak current vs accumulated number of shots for 532 and $1064 \mathrm{~nm}$ triggering.
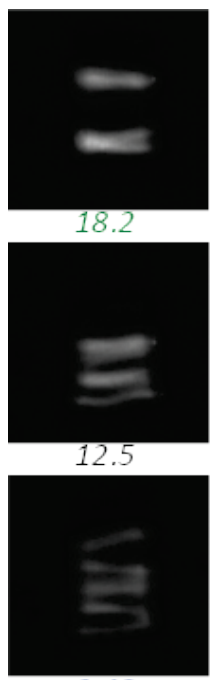

9.48
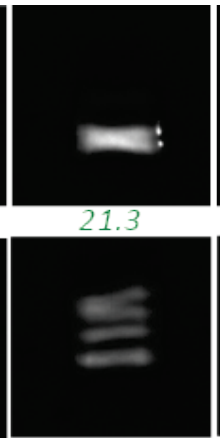

11.8

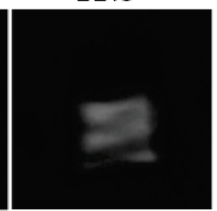

12.4
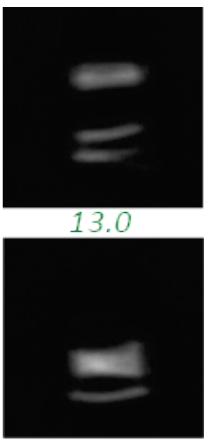

18.2

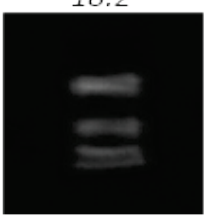

12.6
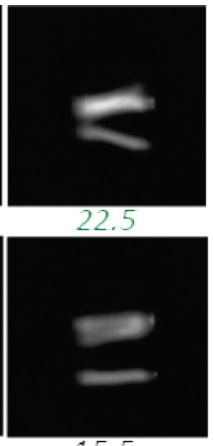

15.5

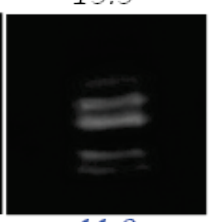

Figure 12. PCSS filaments at 5,50 , and $100 \mu \mathrm{J}$ trigger energies show mean peak/average brightness ratios of $18.78,14.5$, and 11.4 (top to bottom). Reduced peak current is inferred from the peak image brightness.

The peak brightness is reduced by almost 40 percent at $100 \mathrm{uJ}$ compared to $5-10 \mathrm{uJ}$ for the same total current. This indication of reduction in spatial current density accounts for improved lifetime performance. Figure 13 shows the peak current switched using the same parameters as the wavelength lifetest study, using the $1064 \mathrm{~nm}$ triggering, but using trigger energies of $10 \mathrm{uJ}$ (the onset of consistent reliable triggering), $50 \mathrm{uJ}$ (nominal value), and $100 \mathrm{uJ}$. The measurement shows a clear lifetime dependence on the trigger energy, with $>3 \mathrm{x}$ lifetime at $100 \mathrm{uJ}$ vs. $10 \mathrm{uJ}$. 


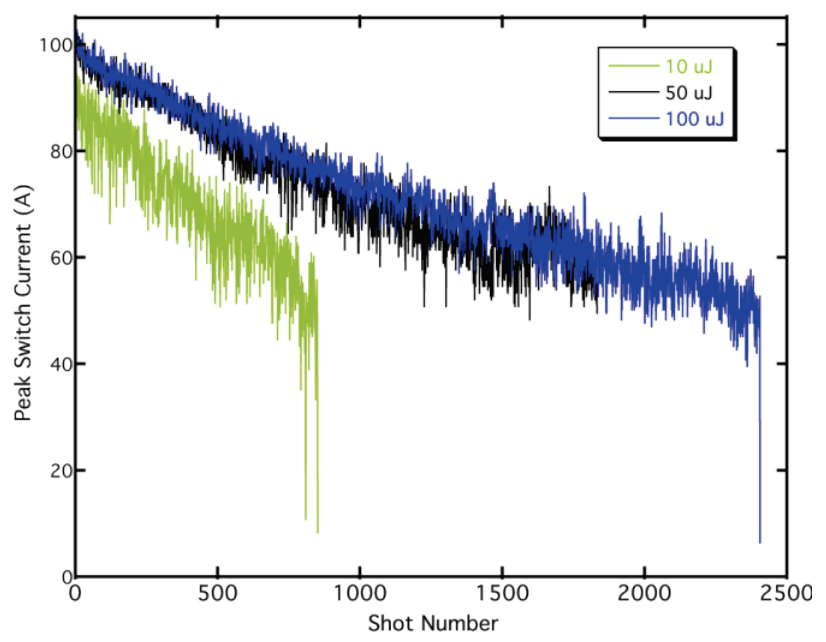

Figure 13. PCSS lifetest -peak current vs accumulated number of shots for 10,50 , and $100 \mathrm{uJ}$ trigger energy.

\section{DEVICE MODELING}

The primary theoretical approach to understanding the performance of these devices involves the solution of the Boltzmann equation. This equation is solved by using an ensemble Monte Carlo method [5]. In this method, an ensemble of electrons and holes flow in momentum space in response to the force exerted by the electric field. During this flow, these particles are scattered by phonons, impact ionization, and Auger recombination. Fig. 14 shows the computed distribution function of electrons for an applied field of $10 \mathrm{kV} / \mathrm{cm}$ in GaAs. This distribution function is used for insight about heating at contacts caused by Auger recombination.

Figure 15 shows the amount of heating at the two contacts in a qualitative manner. At the n-contact (cathode), the holes produce heating given by the steady state energy. This energy is a balance between the heating by the field and the cooling by phonons. However, the heating at the p-contact (anode) has an additional contribution. This contribution is a consequence of the GaAs band structure. As shown in the figure, the lowest GaAs conduction band has more curvature, a lighter mass, than the next energy bands. As a consequence, the electric field heats the electrons to the heavy mass bands. This extra heating is a consequence of phonon cooling that is less effective for lighter mass bands.

A consequence of the additional heating of the electrons is that more energy is released by electrons when they are collected at the p-contact. This additional energy is approximately $0.35 \mathrm{eV}$ for each electron. This extra heating may help explain the larger degradation usually seen at the anodes of these devices. Further work may reveal ways to mitigate the effects of extra heating.

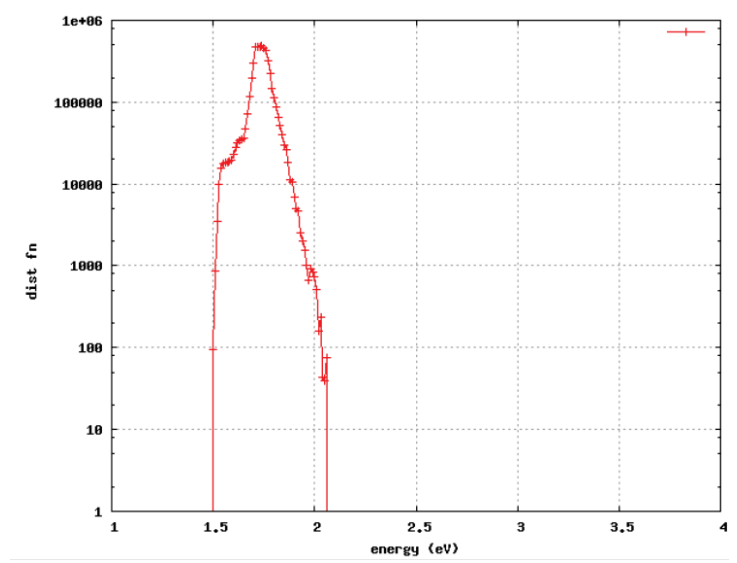

Figure 14. The electron distribution function in GaAs for a $10 \mathrm{kV} / \mathrm{cm}$ applied field.

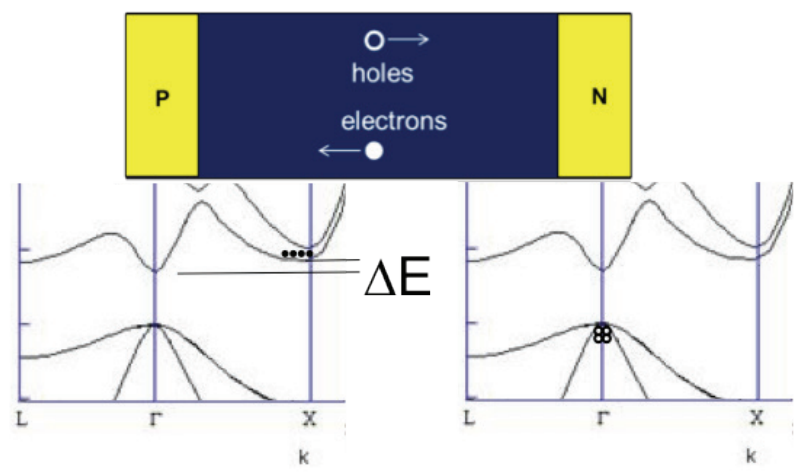

Figure 15. GaAs band structure shows electrons in the next band have more energy to dissipate at the p-contact.

\section{VIII.CONCLUSIONS}

Advances in high current, high gain GaAs PCSS are being made by developing methods to trigger MCSF and improve device contacts. LOS optics with $\mu$-lens arrays, optical masks, and etched ridges and valleys are being tested to improve current sharing and increase filament density. Advanced patterning techniques are being used to reduce high electric field current density regions near the contacts. The effects of wavelength and trigger energy on device lifetime and filament brightness were measured. Recent ideas to improve modeling and simulation of the PCSS lock-on field have led to an explanation for contact lifetime performance.

\section{REFERENCES}

[1] S.F. Glover, et al. "Pulsed and DC Charged PCSS-based Trigger Generators," Proc. 16th IEEE Pulsed Power Conference, Washington, DC, June 2009, pp. 1444-1447

[2] F.J. Zutavern, et al., "Final Report of LDRD Project: A Compact, Repetitive Accelerator...," SLN SAND98-0898, Albuq., NM, 1998.

[3] F.J. Zutavern, et al., "High Current, Multi-Filament PCSS," Proc. 17th IEEE Pulsed Power Conference, Chicago, June 2011.

[4] H.P. Hjalmarson, et al., "Electrical Breakdown Phenomena Involving Material Interfaces," Proc. 18th IEEE Pulsed Power Conference, San Francisco, June 2013.

[5] K E. Kambour, Thesis, Texas Tech, 2003, unpublished. 